\title{
Case Report on Sertoli Cell Tumor of Ovary
}

\author{
KOHINOOR BEGUM ${ }^{1}$, KAMILARA KHANOM ${ }^{2}$, JOYSREE SAHA ${ }^{3}$
}

\begin{abstract}
:
A 33 years old lady presented with history of irregular menstrual cycle followed by menorrhagia. USG revealed left ovarian solid mass but all tumor markers were within normal range. A solid mass on left sided ovary was found on laparotomy. Histopathological examination of the mass showed well differentiated sertoli cell tumor.

Tumors of the stroma (Leydig cells) and/or sex cords (Sertoli cells) represent approximately $8 \%$ of ovarian tumors and develop from the connective tissue (respectively, interstitial and nurse cells) of the ovary. Because these cells participate in ovarian hormonal function, most of the sex-cord or stromal tumors are able to secrete hormones (estrogens, androgens, corticoids), which explains the hormonal dysfunctions associated with these tumors. Their prognosis are difficult to establish; some of the tumors are almost always benign (Sertoli cell tumors, Leydig cell tumors), whereas others are malignant but with more-or-less delayed local-regional or metastatic relapses.
\end{abstract}

Key words: ovarian tumor, sex cord stromal tumor, sertoli cell tumor.

\section{Introduction:}

Ovarian sex cord-stromal tumors are rare, comprising only 1.2 percent of all primary ovarian cancers. ${ }^{1}$ Yet many of these tumors may mimic a range of more common epithelial and germ cell tumors, both benign and malignant. ${ }^{1}$ Two types growth pattern in ovarian tumors that are problematic in terms of overlap between sex cord-stromal, epithelial and germ cell tumors are; 1) tubuloglandular growth and 2) sex cordlike growth. A third but much less common pattern is that of single infiltrative clusters or cells with signet ring features or vacuoles.

Patient's age, clinical features and gross findings are useful for diagnosis. Most patients with ovarian sex cord stromal tumors are young, have hormonal alterations (estrogenic or androgenic manifestations), elevated serum markers (e.g. AFP) or syndromic associations (e.g. Peutz Jeghers syndrome); the tumors are often unilateral, yellow/orange, and multicystic. In contrast, many of the mimics, particularly adenocarcinoma, would be unusual in a young patient and would not likely present with such clinical or gross features ${ }^{2}$. Thus, discordance between the clinical context and the pathologic impression should prompt for further confirmation of the pathologic diagnosis. Generally, we prefer to confirm the morphologic diagnosis of granulosa cell tumor, Sertoli cell tumor or Sertoli Leydig cell tumor with immunohistochemistry given the potential for overlap with so many other tumor types.

\section{Case Report:}

Mrs Rina, 33 years of age hailing from Noakhali, para2 , has had regularly menstruation with average flow and duration developed irregular and infrequent menstruation for the last one year. Her cycle was occurring at 2-3 months interval, persist for 2-3 days but not associated with dysmenorrhoea. With these complaints she attended a local physician and was treated with cyclical progesterone for 7-8 cycles. With this regimen her menstruation becomes regular, but for the last 3 months she noticed excessive per vaginal bleeding during her menstruation. She had no history of weight gain, excessive hair growth or any change of voice.

Regarding contraceptive history- her husband was living abroad and she used OCP occasionally. Per abdominal examination revealed no abnormality.

\section{Per vaginal examination:}

Cervix - healthy, a small polyp came out from endocervix.

1. Professor and Head of the Department, Department of Obstetrics and Gynaecology, Popular Medical College

2. Associate Professor, Department of Obstetrics and Gynaecology, Popular Medical College

3. Assistant Professor, Department of Obstetrics and Gynaecology, Popular Medical College 
Uterus - Bulky, anteverted.

Fornix-free.

USG of lower abdomen was done which revealed left ovarian solid mass of $6.6 \times 5 \mathrm{~cm}$ in size and right ovary normal size. ( Fig-1)

CA-125 was $28.5 \mathrm{U} / \mathrm{ml}$.

Decision was taken to do laparotomy. Before laparotomy diagnostic $D \& C$, endometrial, cervical biopsy and polypectomy was done and polyp with endometrial and cervical tissue were sent for Histopathology.

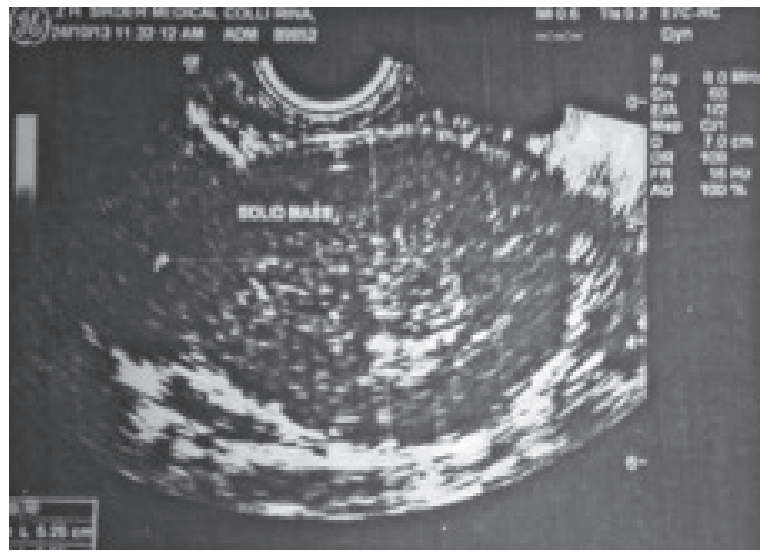

Fig.-1: USG Findings of Ovarian Tumor

On laparotomy, uterus was found 8 weeks pregnancy size, which was freely mobile. Left sided ovarian solid mass with intact capsule and free of adhesion was found. Left tube was inflamed. Left salpingoophorectomy was done \& sent for Histopathology. (Fig-2,3).

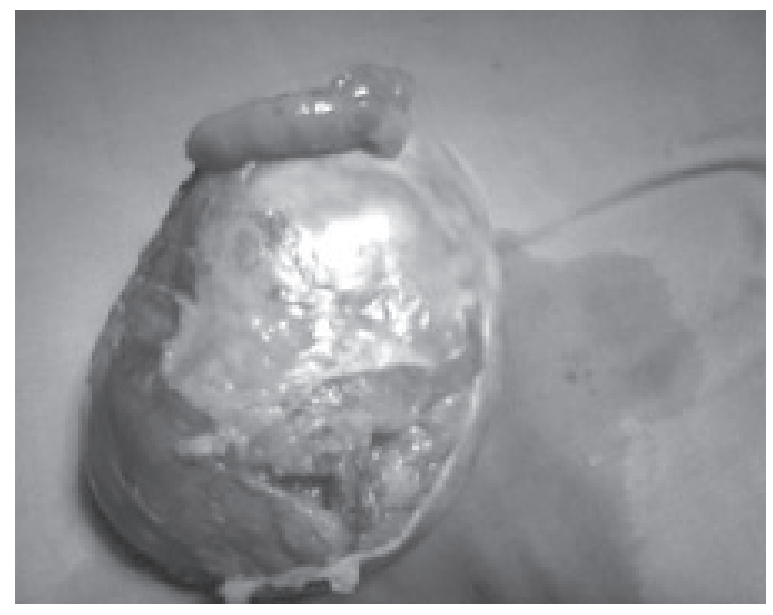

Fig.-2: Gross Picture of Sertoli Cell Tumor

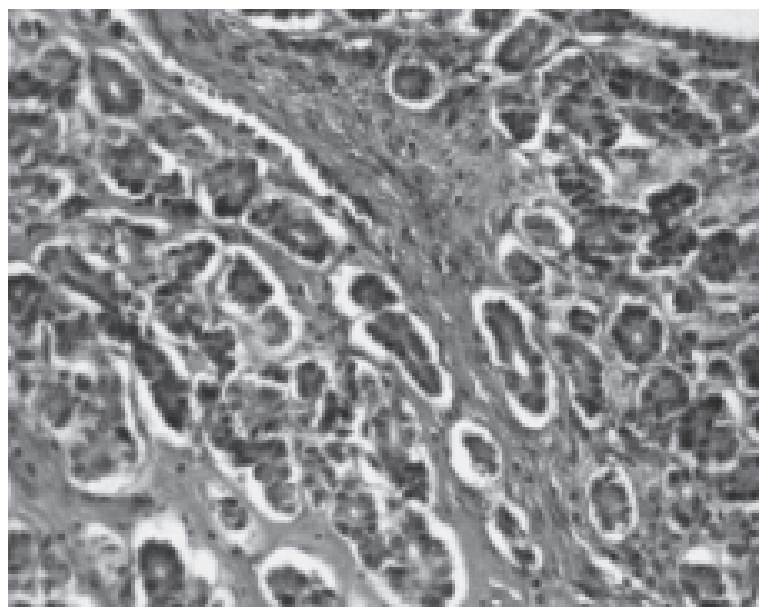

Fig.-3: Well Differentiated Sertoli Cell Tumor. Hollow And Solid Round to Elongated tubules Composed of Sertoli Cells are in a Paucicellular Collagenous Stroma.

Right tube and ovary were apparently healthy and preserved. Liver, kidney, peritoneum, omentum all are found normal.

Histopathology report reveals-

Endometrium- late secretory phase.

Cervix - chronic cervicitis, Endocervical polyp.

Left ovary - sertoli cell tumor well differentiated.

Her postoperative period was unremarkable \& patient was discharged after recovery.

\section{Discussion:}

The application of immunocytochemistry to ovarian tumors has allowed for the placement of a larger spectrum of tumors in the Sertoli cell tumor category, some of which exhibit aggressive behavior. Aseries of 54 ovarian Sertoli cell tumors has been reported. ${ }^{3}$ The tumors typically occur in young patients with a mean age of 30 years, but there is a wide age range. The tumors are more commonly estrogenic than androgenic. Three patients with well differentiated Sertoli cell tumors developed hypertension because of renin secretion by the tumor. ${ }^{3}$ In $11 \%$ of cases, the patient had the Peutz-Jeghers syndrome. The tumors were positive for inhibin in $82 \%$ of cases, and all were negative for epithelial membrane antigen and chromogranin. Inhibin, epithelial membrane antigen, and chromogranin are the most useful immunostains in the differential diagnosis to exclude carcinoid and endometrioid carcinoma. ${ }^{3}$ Wolffian adnexal tumor may resemble Sertoli cell tumor; however, in addition to a solid tubular pattern, the former is characterized by 
an admixture of other histologic patterns, including cysts with a sieve-like appearance that are distinctive and rule out Sertoli cell tumor. Other primary neoplasms that rarely enter the differential diagnosis of Sertoli cell tumor include steroid cell tumor, struma ovarii, and dysgerminoma. ${ }^{3}$ Metastatic neoplasms, particularly Krukenberg tumor, can be confused with Sertoli cell tumor. The presence of an extraovarian primary, bilateral involvement and the microscopic identification of signet-ring cells and marked nuclear atypia suggest the diagnosis of Krukenberg tumor.

The topic of Sertoli cell tumors and its relationship to sex cord tumor with annular tubules is arguably the most controversial aspect of the classification of ovarian sex cord stromal tumors. Although the preponderance of evidence suggests that the sex cord tumor with annular tubules shows Sertoli cell differentiation. ${ }^{4}$ Most notable authorities believe that, because of its unique morphological and clinical features, this neoplasm should be classified separately. The third WHO classification allows for its inclusion either as a Sertoli cell tumor (Sertoli cell tumor, complex tubular type) or separately as sex cord tumor with annular tubules. ${ }^{5}$ It is possible that molecular studies will resolve this issue in the future.

A number of variants of ovarian Sertoli cell tumor have been described including typical, lipid-rich, and oxyphilic forms. ${ }^{3}$ To be considered a variant, aneoplasm should be composed entirely or predominantly $(>50 \%)$ of that pattern. The dominant microscopic pattern of typical (not otherwise specified) Sertoli cell tumors is tubular (Fig. 3), but cordlike and diffuse patterns are common, and rarely, alveolar and retiform patterns are encountered. ${ }^{6}$ Those who consider the sex cord tumor with annular tubules to be a variant of Sertoli cell tumor would also include a complex tubular type. ${ }^{4}$ It is of considerable significance that, in addition to the sex cord tumor with annular tubules (complex tubular type), all of the forms of Sertoli cell tumor that occur in the ovary, the typical, lipid-rich, and oxyphilic types have been associated with the Peutz-Jeghers syndrome. Two additional variants that occur in the testis, the large cell calcifying and sclerosing types, are almost nonexistent in the ovary. ${ }^{6}$ Sertoli cell tumors have a stroma that varies from negligible, to delicate fibrous septa, to conspicuous and hyalinized. The stroma may contain rare cells consistent with Leydig cells. If more than rare Leydig cells are present, the diagnosis of well-differentiated Sertoli-Leydig cell tumor should be rendered. Well-differentiated Sertoli cell tumors are almost always benign, whereas those exhibiting atypical features frequently present with advanced stage disease and exhibit aggressive behavior. ${ }^{3,6}$ The histologic features of ovarian Sertoli cell tumors that best correlate with adverse outcome are nuclear atypia, 5 or more mitotic figures per 10 high power fields, and tumor necrosis. ${ }^{3}$ Bizarre nuclei of degenerative type unassociated with an elevated mitotic index do not seem to adversely influence prognosis. $^{1}$

\section{Prognosis:}

The incidence of clinical malignancy in these tumors is $10-30 \%$. Patients with early stage experience recurrences. Eleven percent of tumors with intermediate differentiation and $60 \%$ of poorly differentiated tumors are likely to progress clinically. Tumors which show extraovarian spread or metastases at the time of laparotomy usually are fatal. Recurrence or metastases in early stage disease usually become apparent within twelve months, often before six months and are often preceded by an exacerbation of the patient's vilrilizations. Metastases have been in the omentum, abdominal lymph nodes or liver and less often in lungs, bone, intestine, kidney, mediastinum or brain.

\section{Conclusion:}

Sertoli cell tumors are uncommon and usually present at reproductive age with estrogenic effect. Almost all tumors are solid and unilateral.

\section{References:}

1. Young RH. Sex cord-stromal tumors of the ovary and testis: their similarities and differences with consideration of selected problems. Mod Pathol 2005; 18 Suppl 2:S81.

2. Vijayaraghavan M. Sex cord-stromal tumors: pathological considerations. Indian journal of Medical \& Paediatric oncology. 2004;25(1):32-37.

3. Oliva E, Alvarez T, Young RH. Sertoli cell tumors of the ovary: a clinicopathologic study immunohistochemical study of 54 cases. Am J Surg Pathol. 2005;29:143-56.

4. Tavassoli FA, Norris HJ. Sertoli tumors of the ovary. A clinicopathologic study of 28 cases with ultrastructural observations. Cancer 1980;46:2281-97.

5. Tavassoli FA, Devilee P. (eds). Pathology and Genetics of Tumours of the Breast and Female Genital Tract. $3^{\text {rd }}$ edition. IARC Press. Lyon 2003

6. Young RH. Sex cord-stromal tumors of the ovary and testis; their similarities and differences with consideration of selected problems. Mod Pathol 2005;18:s81-s98. 